\title{
Plataformas virtuales y su impacto en la Educación Superior.
}

\section{Virtual platforms and their impact on Higher Education.}

\author{
Pablo Homero Velasteguí ${ }^{1}$
}

\section{Resumen.}

La educación en la actualidad es la encargada de no solo transmitir conocimientos e información, sino que debe proporcionar los medios, aptitudes y habilidades para producirlos y utilizarlos en la práctica pedagógica, creando nuevos escenarios donde desarrollan el proceso educativo.

Una de las vías fundamentales para el desarrollo de tales propósitos es la utilización de las Aulas Virtuales, como apoyo al docente permiten elevar el desempeño y el trabajo colaborativo, a través de la interactividad y la comunicación entre el docente y el estudiante, aprovechando las facilidades que brindan los productos multimedia para la presentación de contenidos.

Las aulas virtuales como espacio educativo se han convertido en un medio muy utilizado en las universidades, a través de ellas los docentes y estudiantes participan en un proceso de enseñanza aprendizaje mediante el empleo efectivo de las computadoras para acceder, comunicar, compartir y crear conocimientos que conducen al aprendizaje.

La adecuación de tales prácticas tecnológicas en el ámbito educativo promueve la creación de nuevos entornos didácticos que afectan de manera directa tanto a los actores del proceso de enseñanza - aprendizaje como al escenario donde se lleva a cabo el mismo.

Son un nuevo ámbito de aprendizaje, una poderosa herramienta de comunicación, información e interacción entre los docentes y los estudiantes, trascienden las barreras de espacio y tiempo, permiten una comunicación directa y atención personalizada inmediata o diferida entre docente y estudiantes, brindan una variedad de recursos que promueven el proceso de enseñanza - aprendizaje colaborativo y la construcción de nuevos conocimientos a través de ellas.

\footnotetext{
${ }^{1}$ Ciencia digital, Ambato, Ecuador, luisefrainvelastegui@ cienciadigital.org
} 
La educación virtual es una nueva forma viable de enseñanza que viene a suplir necesidades, precariedades propias de la educación presencial y del mal uso de la tecnología educativa.

Palabras claves: Tecnologías de la educación, Aulas Virtuales, proceso de enseñanza aprendizaje, tecnologías de la información y la comunicación.

\section{Abstract.}

Education today is responsible for not only transmitting knowledge and information, but must provide the means, skills and abilities to produce and use them in pedagogical practice, creating new scenarios where they develop the educational process.

One of the fundamental ways for the development of such purposes is the use of Virtual Classrooms, as a support to the teacher they allow to elevate the performance and the collaborative work, through the interactivity and the communication between the teacher and the student, taking advantage of the facilities that provide multimedia products for content presentation.

Virtual classrooms as an educational space have become a widely used medium in universities, through which teachers and students participate in a teaching-learning process through the effective use of computers to access, communicate, share and create knowledge that lead to learning.

The adaptation of such technological practices in the educational field promotes the creation of new didactic environments that directly affect both the actors of the teaching - learning process and the scenario where it takes place.

They are a new learning environment, a powerful tool for communication, information and interaction between teachers and students, transcend the barriers of space and time, allow direct communication and immediate or delayed personalized attention between teacher and students, provide a variety of resources that promote the teaching process collaborative learning and the construction of new knowledge through them.

Virtual education is a viable new form of education that comes to meet the needs, precariousness of face-to-face education and the misuse of educational technology.

Keywords: Educational technologies, Virtual Classrooms, teaching - learning process, information and communication technologies.

\section{Introducción}

Las aulas virtuales y su importancia en la educación superior

Imagen N.1: Las Aulas Virtuales 


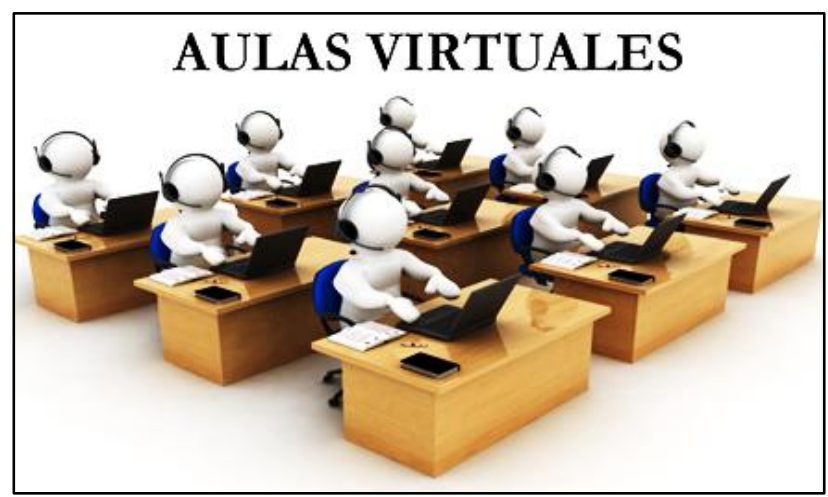

Fuente: http://aulasvirtualesenlaeducacion.blogspot.com/2015/08/que-es-aulavirtual.html

\section{Elaborado por: María Lindao}

Las Aulas Virtuales en varias investigaciones teóricas realizadas de (Abrigo, 2015; Chamba, 2015 y Delgado, 2009) afirman que el Aula Virtual es un espacio caracterizado por la innnovacion educativa, el énfasis en la actividad sobre los contenidos, la participacion creativa de los alumnos y el aprendizaje colaborativo que se realiza mediante el uso de elementos tecnológicos.

La educación virtual es una nueva forma viable de enseñanza que viene a suplir necesidades, precariedades propias de la educación presencial, de saberes y de prácticas educativas mediante soportes virtuales, sin barreras de tiempo y distancia, que permite la construcción de un gran campus virtual a nivel planetario, sustentando en los mecanismos de la interactividad e interconectividad que se desprenden de la incorporación de las tecnologías en el campo educativo.

Las Aulas Virtuales en la educación según la investigación realizada de (Porter, 1991) define que la evolución de las Plataformas educativas se muestra muy ligada al desarrollo de la sociedad de la información y del conocimiento, y más en concreto, al de los sistemas educativos, que tienden, progresivamente, a adaptarse a las necesidades reales del mundo laboral.

En este marco de innovación y cambio, la opción de generar entornos virtuales de aprendizaje basados en las Tecnologías de la Información y la Comunicación (TIC), supone responder de forma integral los requerimientos que impone la Sociedad del Conocimiento y a las nuevas necesidades del entorno educativo. Es dentro de este contexto de innovación donde surgen las plataformas educativas virtuales.

La educación actual afronta múltiples retos y uno de ellos es dar respuestas a los profundos cambios sociales, económicos y culturales que se prevén para la llamada "Sociedad de la Información y el Conocimiento" en esta era de la información y la comunicación, siendo el internet el que ha generado un enorme interés en todos los ámbitos de nuestra sociedad y gracias a su creciente uso con fines educativos se ha convertido en un campo abierto a la reflexión e investigación. 
Sabiendo que las Aulas Virtuales son un medio en que los educadores y educando se encuentran para realizar actividades que conducen al aprendizaje, y que el concepto de conocimiento es la capacidad para transformar datos e informaciones en acciones efectivas y eficaces.

La modalidad de formación por medio de las Aulas Virtuales, ha mostrado mayor efectividad, despedazando las barreras de tiempo, al brindar técnicas, métodos y recursos que crean mas efectivo y flexible el proceso de enseñanza - aprendizaje, ya que consienten el uso de las tecnologías de información y comunicación.

Las Aulas Virtuales brindan al estudiante y docente la oportunidad de obtener nuevos conocimientos mediante su manipulación a través del proceso de enseñanza aprendizaje, son medios que permiten desarrollar diferentes actividades académicas y de investigación encontrando en este entorno una comunidad propia para realizar, orientar nuevos conocimientos a través de la comunicación y distribución.

\section{Factores de importancia en la Educación Virtual}

Los factores que se consideran importantes segun las investigaciones obtenidas de (Bernad, 2016; Camacho, 2016 y Machado, 2004) afirman que son los siguientes:

$\checkmark$ Exploracion: El uso de Internet como una herraienta de exploración les abre al profesor y al estudiante univesitario las puertas de una fuente inagotable de informacion y recursos.

$\checkmark$ Experienca: El estudiante cirtual se ve involucrado en una nueva experiencia social y de aprendizaje que puede incluir comunicaciones directas con su profesor, discusiones con sus compañeros de curso o estudio individual de contenidos a su propio ritmo.

$\checkmark$ Compromiso: Los recursos virtuales ofrecen una oportunidad unica al estudiante de comprtir experiencias con otros, lo que refuerza el sentido de colaboracion y de comunidad. Ademas el estudiante recibe el control de su tiempo y sus recursos, puede escoger el mejor camino de aprendizaje de acuerdo con sus preferencias y capacidades.

$\checkmark$ Flexibilidad: Desde cualquier lugar y a cualquier hora, los estudiantes pueden tener acceso a sus cursos virtuales. Se estima que aproximadamente $80 \%$ de las empresas ya tienen solucionado su acceso a Internet, por lo que los obstáculos de acceso de los estudiantes que trabajan ya estan superados.

Las aulas virtuales son muy importantes en la educación, se encuentra todos los factores que existen en el aula, este entorno educativo, permite al estudiante realizar sus trabajos en linea de forma independiente, así como las oportunidades de interacción que normalmente existen en el aula y en la enseñanza cara a cara con el docente y posibilita el desarrollo del proceso de aprendizaje aplicando las tecnologías de la información y comunicación TICs.

Las Aulas Virtuales les permiten a los estudiantes e instructores comunicarse utilizando funciones como audio, video, chat de texto, pizarra interactiva y uso compartido de 
aplicaciones. El propósito del estudio adopta aulas virtuales sincrónicas y los apéndices especiales ofrecen información actualizada sobre grupo de noticias y listas de correos, sitios web de instrucción, recursos en línea para subvenciones y propuestas, y muchos más.

\section{El modelo educativo en línea}

Imagen N.2: El Modelo Educativo en Línea

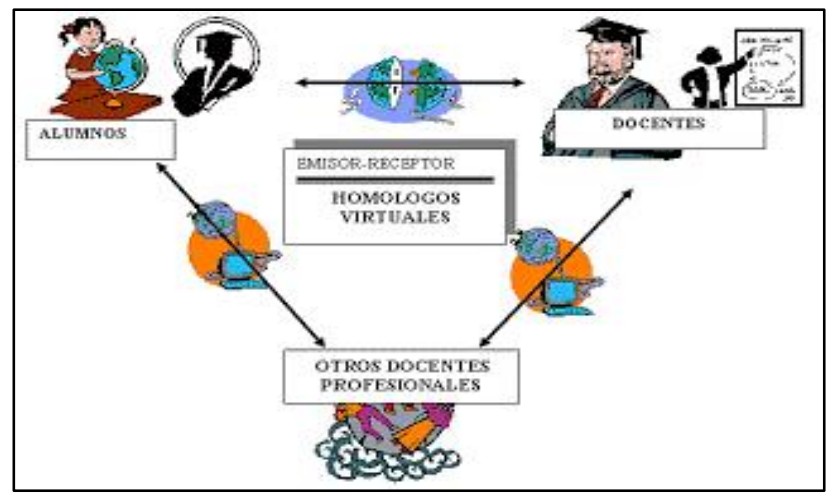

Fuente: http://educacionvirtual3.blogspot.com/

Elaborado por: Rocío Montes

El modelo educativo en línea en varias fuentes bibliográficas realizadas de (Aretio, 1994; Domínguez, 2013; Khvilon, 2003 y Lepez, 2014) afirman que la educación virtual el aprendizaje está centrado en el alumno y su participación activa en la construción de conocimientos le asegura un aprendizaje significativo. Con esto, se definen los contenidos y actividades para un curso partiendo de la estrategia didáctica diseñada por el docente. El estudiante universitario realiza su proceso de aprendizaje a partir de dichos contenidos y actividades, pero sobre todo, a través de su propia motivación por aprender, de la interacción con otros compañeros y de la guía y asesoría de su docente.

El estudiante universitario mediante las plataformas virtuales aprende de forma más activa y dinámica ya que no sólo recibe la instrucción del docente, sino que aprende a través de la búsqueda de información, la autorreflexión, analizando cada concepto investigado y obteniendo nuevos conocimientos al momento de realizar las diversas actividades de manera individual en el proceso de la enseñanza - aprendizaje.

A través de estas herramientas tecnológicas la educación se ha convertido más facil ya que el docente y estudiante pueden estar conectados mediante el internet, para realizar sus trabajos, evaluaciones, investigar, etc., sin duda permite enriquecer obteniendo nuevos conocimientos al manipular estas tecnologías virtuales. 
Tabla N.1: Ventajas y desventajas de la enseñanza virtual en la educación superior

\begin{tabular}{|c|c|}
\hline VENTAJAS & DESVENTAJAS \\
\hline $\begin{array}{c}\text { Se siente personalizado en el trato con el } \\
\text { docente y sus compañeros }\end{array}$ & $\begin{array}{c}\text { Inasistencia de estructura pedagógica en } \\
\text { la ifnromación y multimedia. }\end{array}$ \\
\hline $\begin{array}{c}\text { Puede adaptar el estudio a su horario } \\
\text { personal, teniendo acceso a la enseñanza, } \\
\text { no viéndose perjudicados aquellos que no } \\
\text { pueden acudir periodicamente a clase por } \\
\text { motivos de trabajo o distancia, etc. }\end{array}$ & $\begin{array}{c}\text { Dificultades organizativas, problemas } \\
\text { técnicos y altos costos de } \\
\text { mantenimiento. }\end{array}$ \\
\hline $\begin{array}{c}\text { Puede realizar sus participaciones de } \\
\text { forma meditada gracias a la posibilidad } \\
\text { dee trabajar off-line. }\end{array}$ & $\begin{array}{l}\text { Temor a que los estudiantes vean los } \\
\text { medios con la pasividad con que se mira } \\
\text { un programa de televisión, } \\
\text { caracterizado por una tendencia al } \\
\text { facilismo inmediato, inconveniente para } \\
\text { aprender ciertos contenidos. }\end{array}$ \\
\hline $\begin{array}{c}\text { Podrá seguir el ritmo de trabajo marcado } \\
\text { por el docente y por sus compañeros de } \\
\text { curso. }\end{array}$ & $\begin{array}{l}\text { La tendencia a trabajr cualquier aspecto } \\
\text { o contenido de forma virtual, dejando } \\
\text { de lado el uso de medios más sencillos } \\
\text { como retroproyector. }\end{array}$ \\
\hline $\begin{array}{l}\text { El estudiante tiene un papel activo, que } \\
\text { no se limita recibir información sino que } \\
\text { forma parte de su propia formación. }\end{array}$ & $\begin{array}{c}\text { Falta de una estructura pedagógica } \\
\text { adecuada y diseñada en los procesos } \\
\text { cognitivos y las formas de aprender de } \\
\text { los estudiantes. }\end{array}$ \\
\hline $\begin{array}{l}\text { Existe el feed-back de información, de } \\
\text { manera que el docente conoce si el } \\
\text { estudiante responsable al método y } \\
\text { alcanza los objetivos fijados inicialmente. }\end{array}$ & $\begin{array}{c}\text { Una mala conexión de internet, } \\
\text { falencias en el equipo o material de } \\
\text { trabajo pueden generar retrasos e } \\
\text { interrupciones. }\end{array}$ \\
\hline $\begin{array}{l}\text { Se beneficia de las ventajas de los } \\
\text { distintos métodos de enseñanza y medios } \\
\text { didácticos tradicionales, evitando los } \\
\text { inconvenientes de los mismos. }\end{array}$ & $\begin{array}{l}\text { Sin una rutina de estudios programada, } \\
\text { se puede descontrolar los horarios para } \\
\text { otras actividades. }\end{array}$ \\
\hline
\end{tabular}

Fuente: Investigada

Elaborado por: Ruth Calo

A pesar de las múltiples ventajas que ofrece el recurso virtual no se pueden desconocer los riesgos potenciales por el mal uso que se le puede dar, a traves de su uso. Mediante el modelo educativo el docente diseña la estrategia didáctica que va transmitir al estudiante, el mismo que le permite su concentración y participación activa dentro del aula de clases y asi construir nuevos conocimientos para un mejor aprendizaje. 
El plan de estudios centra su atención en el desempeño de los estudiantes a través del uso de nuevos conocimientos, actitudes y habilidades ante la actividad o solución de un problema. El estudiante de la educación virtual aprende de forma más activa pues no sólo recibe la instrucción del docente, sino que aprende a través de la búsqueda de información, la autorreflexión y las diversas actividades que realiza de manera individual y colaborativa.

\section{Educación virtual}

La educación virtual en varias fuentes investigadas de (Gallo, 2013; Gómez, 2011 y Moreno, 2012) definen que las nuevas tecnologías de la información y comunicación posibilitan la creación de un nuevo espacio social - virtual para las interrelaciones humanas, este nuevo entorno, se está desarrollando en el área de la educación, porque posibilita nuevos procesos de aprendizaje y transmisión del conocimiento a través de las redes modernas de comunicaciones.

El espacio virtual, que le llamo aulas sin paredes, cuyo mejor exponente actual es la red Internet, no es presencial sino representacional, no es próximal, sino distal, no es sincrónico, sino multicrónico, y no se basa en recintos espaciales con interior, frontera y exterior, sino que depende de redes electrónicas cuyos nodos de interacción pueden estar determinados por diversos países.

\section{Mundos virtuales aportan al espacio real.}

Sus caracteristicas son:

$\checkmark$ En la representación para comprender mejor lo real.

$\checkmark$ Son mundos en potencia, que nadie puede prever la magnitud de su avance.

$\checkmark$ Se entremezclan entre lo real y lo virtual, lo potencial y lo actual.

$\checkmark$ Se alimentan de la vida intermedia de los lenguajes simbólicos.

$\checkmark$ Se alimentan de la realidad visible.

$\checkmark$ Son intangibles, de lo sensible, la concepción y la percepción.

$\checkmark$ Son sistemas que procuran darnos la ilusión más convincente posible de inmersión funcional dentro de un mundo sintético.

$\checkmark$ Representa una situación real, la televirtualidad, la teleintervenciones o de telerrobótica, tambiédiscutiblemente reunidas bajo la etiqueta de telepresencia.

$\checkmark$ Se esconde bajo la inconciencia de lo potencial humano.

$\checkmark$ Es parte del potencial aristotélico, aptitud para recibir una forma.

$\checkmark$ La potencia hace que enecuentre vías de actualización, en virtudes necesarias para su determinación.

\section{Los entornos virtuales entes socializadores}

La comunicación educativa refuerza las enormes potencialidades comunicativas que ofrecen los entornos virtuales en cuanto a la socialización, lo paradójico es su acentralidad favorece la desjerarquización de las relaciones humanas. Su ubicuidad fomenta la distribución, intercambio y circulación de información, ideas y conocimientos. Su 
plasticidad permite la aprehensión sensible de modelos conceptuales aparejando un enorme salto el modo de abordad los objetos de conocimiento.

\section{El conocimiento virtual}

$\checkmark$ Surge de la virtud del hombre en elalcance de conocer la verdad y la realidad de las cosas.

$\checkmark$ Es dependiente de las leyes de las redes virtuales, y la reacción personal a partir de la virtud humana.

$\checkmark$ Es dependiente de las leyes de las redes virtuales, y la reacción personal a partir de la virtud humana.

$\checkmark$ Está estructurado de acuerdo a los parámetros de versatilidad.

$\checkmark$ Facilidad de acceso, rapidez de descarga y multimediaticidad.

$\checkmark$ Tieneposibilidad de manipularlo interactuar con cualquier usuario remoto.

$\checkmark$ Se puede generarse un debate, sin tener un tema específico en Internet.

$\checkmark$ Se pude interactuar con individuos de cualquier parte del mundo.

$\checkmark$ No es Internet, sino el saber que existe en Internet.

\section{Educación y virtualidad}

La educación es un ente socializadora que impulsa al conocimiento epistemológgico a sumergirse, hasta llegar el proceso de dialecticidad. Sin embargo, como ya antecedemos, la virtualidad no tiene límites, ni reglas, es espontáneo y transformador que parte de la virtud humana.

En las sociedades se constituyen la diferenciación de las clases. Las clases sociales tiende a estar concentradas regionalmente, la división entre los barrios de las ciudades, sin embargo, el espacio virtual o la virtualización, en cambio aparece como un espacio homogéneo, sin diferencias de clases, razas o sexos. Que integra a grandes masas sociales, sin que tenga este limitaciones a acceso a la educación.

La educación virtual enmarca la utilización de las nuevas tecnologías, hacia el desarrollo de metodologías alternativas para el aprendizaje de alumnos de poblaciones especiales que estan limitadas por su ubicación geográfica, la calidad de docencia y el tiempo disponible.

LA UNESCO define como entornos de aprendizajes que constituyen una forma totalmente nueva en relación con la tecnología educativa, un programa informático interactivo de carácter pedagógico que posee una capacidad de comunicación integrada. Son una innovación relativamennte reciente y fruto de la convergencia de las tecnologías informáticas y de telecomunicaciones que se ha intensificado durante los últimos años.

Por otro lado, la educación virtual como la educación del siglo XXI, tiene los siguientes principios.
$\checkmark$ La autoeducación
$\checkmark$ La autoformación
$\checkmark$ La desterritorrialización 
$\checkmark$ La descentración

$\checkmark$ La virtualización

$\checkmark$ La tecnologización

$\checkmark$ La sociabilidad virtual

La virtualidad no es algo nuevo en la historia de la humanidad. Hoy en día la tecnología nos brinda ese potencial, de posibilidad de incluso, visionarlo con nuestros propios ojos, reconstruir la imaginación de hacer realidad visual nuestras ideas. Se trata de lo que paradójicamente llamamos realidad virtual.

La educación virtual es una estrategia educativa basada en el uso intensivo de las herramientas tecnológicas, estructuras operativas flexibles y métodos pedagógicos, altamente eficientes en el proceso de enseñanza - aprendizaje; que permite que las condiciones de tiempo, espacio, ocupación o edad de los estudiantes no sean factores limitantes o condiciones para el aprendizaje.

\section{Educación virtual, aprendizaje autónomo y construcción de conocimiento.}

Imagen N.3: Aprendizaje Autónomo en la Educación a Distancia

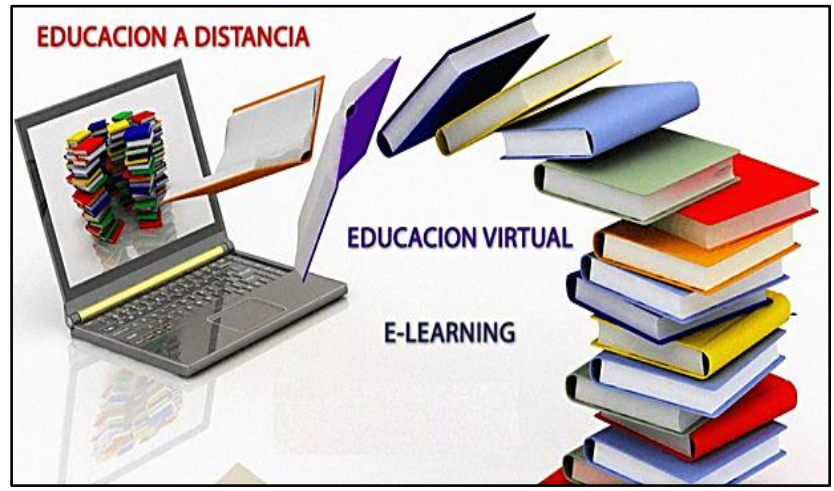

Fuente:https://www.xn-javiercaon-09a.com/2017/10/aprendizaje-autonomoeducacion-distancia-virtual.html

Elaborado por: Javier Cañon

La educación virtual superior en varias investigaciones realizadas de (Delgado, 2010; Sierra, 2012; Vargas, 2004 y Zambrano, 2012) afirman que responden a la necesidad de una nueva visión y modelo de enseñanza - aprendizaje expresada en el marco de una sociedad en permanente cambio que exige replantear la manera de elaborar, adquirir y transmitir conocimiento con criterios de calidad y equidad. Esta clase de educación permite una formación sin limitaciones desde cualquier parte, a toda hora y por cualquier persona, lo que cobra cada vez más valor, sentido y significado en estos tiempos de globalización, información y conocimiento.

Esta modalidad denominada educación virtual se centra en el proceso de enseñanza aprendizaje apoyado por las TIC en una plataforma informática que facilita la interactividad entre alumnos, docentes, compañeros de estudio y materiales multimedia puestos a su alcance en la red Internet. También, constituye un proceso sistemático que implica establecer y definir con claridad los objetivos de aprendizaje, componentes, 
interrelacionales, mecanismos de calidad, procedimientos, seguimiento y control de información.

Sin duda, estos paradigmas de hoy exigen y obligan a nuevas y profundas transformaciones en las dinámicas de enseñanza - aprendizaje de las disciplinas y saberes en la Educación Superior. Estos nuevos retos imponen un enfoque socio constructivista y un modelo más flexible, móvil, pertinente y atónomo centrado más en aprender haciendo que en enseñar, basado en el uso creativo de herramientas y en recursos de formación a través de un aprendizaje colaborativo, significativo, distribuido y dinámico con formas innovadoras e interactivas de trabajo.

Estas nuevas dinámicas implican en un modelo donde los procesos de aprendizaje sean cada vez más eficientes y más efectivos con el fin de contribuir a la contrucción y la divulgación de conocimiento no sólo en su esencia, sino tambien en su aplicación. Por lo tanto, necesita vislumbrar las tendencias, amenazas, cambios y oportunidades que enfrentan los modelos de aprendizaje clásicos que han sido soportados históricamente en un enfoque basado en el acopio y transmisión fragmentada y memorística de información, frente a una nueva formación más amplia, diversa y flexible que constantemente es retada por la rápida incoroporación de nuevas experiencias pedagógicas, didácticas y tecnológicas.

Muchos aspectos de la humanidad que tienen que ver con su propia evolución van cambiando, van mejorando, se van transformando. Se observa como a medida que el tiempo pasa, uno de los aspectos más importantes en la formación de toda persona es su educación. La educación entonces queda también inscrita en ese proceso de cambio, el cual va de la mano con el desarrollo tecnológico que la ciencia nos brinda, es por eso que la presente investigación se abordará lo que se percibe en relación con la evolución que ha tenido la educación.

\section{La calidad en la formación virtual}

Una formación virtual de calidad debe responder a unos requerimientos técnicos y metodológicos que satisfagan las necesidades de los usuarios, es decir que proporcione la posibilidad de consultar materiales didácticos de calidad materiales que le aporten conocimientos y no solo información y medios estables para la comunicación entre docente y estudiante detro del ámbito educativo.

La docencia a nivel superior va desarrollando alternativas para que la enseñanza se vaya transformando, por los nuevos tiempos, exigencias y educación actualizada. Las nuevas exigencias en la educación se centran en la mejora del proceso educativo, y en ese sentido la integración de las TICs facilita aspectos relacionados con la mejora del trabajo individual, la autonomía del alumnado, la facilidad para el desarrollo del trabajoo en equipod u colaborativo, la posibilidad de modificar y adaptar los métodos de evaluación y la interacción bidireccional entre el docente y el estudiante.

La educación superior y el docente han avanzado mucho en la mejora de la educación, pero también es importante que se siga desarrollando hábitos de la utilización de los 
medios tecnológicos, pero con direccionamiento hacia el crecimiento cognitivo, no solo como herramienta de trabajo, sino como dinamizadora de la educación.

La incorporción de las tecnologías de la información y la comunicación en educación superior supone una apuesta por una univesrodad realmente competitiva que trata de potenciar el aprendizaje permanente, lo que va incidir poderosamente en el fortalecimiento de la calidad y en la expansión de las universidades a distancia.

Realmente con la ayuda de las TICs se obtiene una educación competitiva basad en la calidad del buen uso de los docentes y de los estudiantes, marcando una progresión sistemática con resultados muy ambicioso que justamente evoluciona positivamente en los procesos en la enseñanza - aprendizaje.

La educación virtual se centra en el ámbito educativo facilitando la interactividad y conectividad entre estudiantes y docentes, compañeros de clase y grupos de trabajo. Es una estrategia educativa que permite la aplicación de nuevos métodos enfocados al desarrollo del aprendizaje, los cuales están centrados en el estudiante y en la participación activa.

Cual es la frecuencia del uso de las herramientas de una plataforma educativa en el año 2009.

Tabla N.2: Variable, frecuencia y porcentaje.

\begin{tabular}{|l|c|c|}
\hline \multicolumn{1}{|c|}{ Variable } & Frecuencia & Porcentaje \\
\hline Alto & 60 & $60 \%$ \\
\hline Medio & 20 & $20 \%$ \\
\hline Bajo & 10 & $10 \%$ \\
\hline Regular & 10 & $10 \%$ \\
\hline Total & $\mathbf{1 0 0}$ & $\mathbf{1 0 0 \%}$ \\
\hline
\end{tabular}

Fuente: http://plataformas-educativas.blogspot.com/

Elaborado por: Ruth Calo

Gráfico N.1: Uso de las herramientas de una plataforma educativa. 


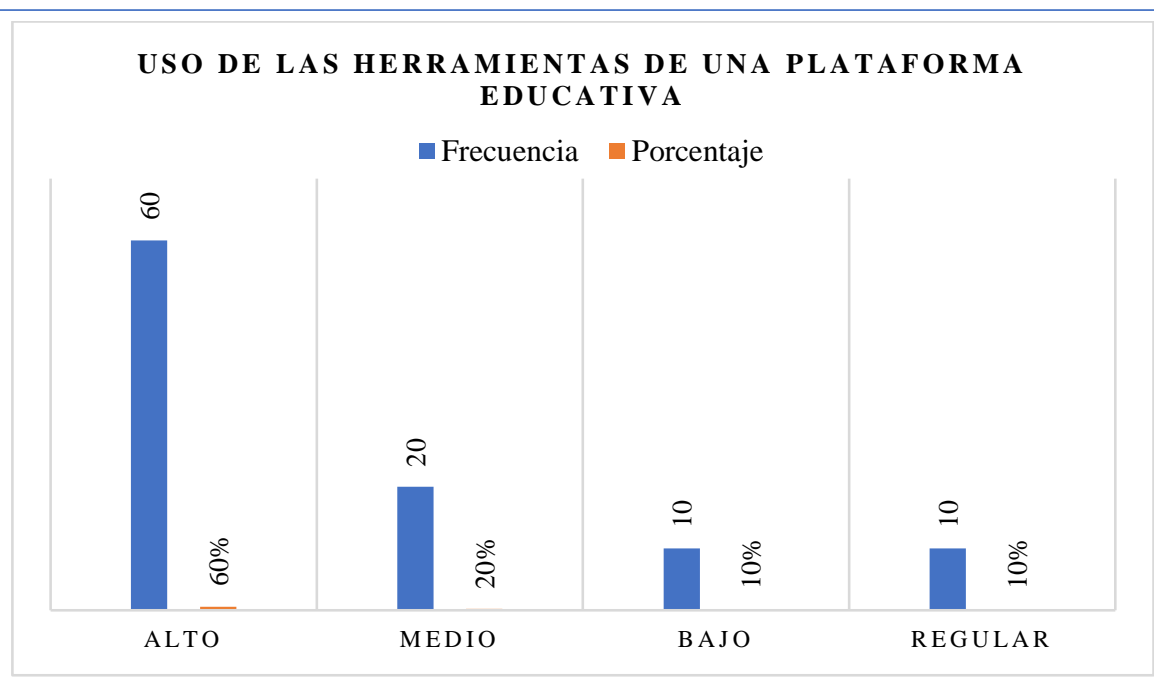

Fuente: http://plataformas-educativas.blogspot.com/

Elaborado por: Ruth Calo

Según la investigación realizada en el año 2009, se ha podido observar que el $60 \%$ de las personas usan las herramientas de una plataforma educativa, el $20 \%$ se encuentran en un nivel medio, el $10 \%$ se encuentran en un nivel bajo y el $10 \%$ de las personas usan regularmente estas herramientas tecnológicas.

El nivel de tecnología en la institución de educación superior en el año 2017 es:

Tabla N.3: Variable, frecuencia y porcentaje.

\begin{tabular}{|l|c|c|}
\hline \multicolumn{1}{|c|}{ Variable } & Frecuencia & Porcentaje \\
\hline Alto & 172 & $87 \%$ \\
\hline Medio & 14 & $7 \%$ \\
\hline Bajo & 12 & $6 \%$ \\
\hline Total & $\mathbf{1 9 8}$ & $\mathbf{1 0 0 \%}$ \\
\hline
\end{tabular}

Fuente: https://www.3ciencias.com/wp.content/uploads

Elaborado por: Ruth Calo

Gráfico N.2: El nivel de tecnologia en la educacion superior. 


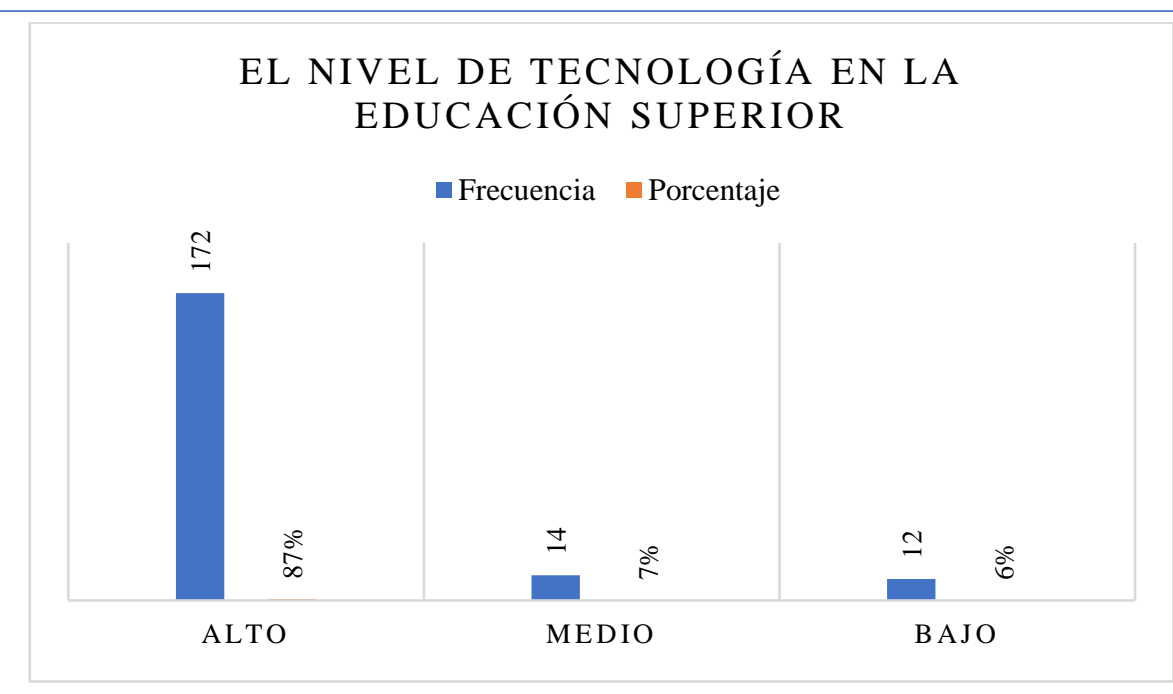

Fuente: https://www.3ciencias.com/wp-content/uploads/2017/03/art3.pdf

Elaborado por: Ruth Calo

En lo que se refiere a la percepción que tienes los docentes del nivel de tecnologías de la IES, un $87 \%$ considera que tiene un nivel alto, el $7 \%$ nivel medio y el $6 \%$ nivel bajo. Lo cual muestra que si existen los medios tecnológicos necesarios para impartir una educación de calidad permitiendo enfrentar los desafíos del proceso enseñanza aprendizaje dentro de las aulas de clases.

Gráfico N.3. Utilidad de las plataformas educativas

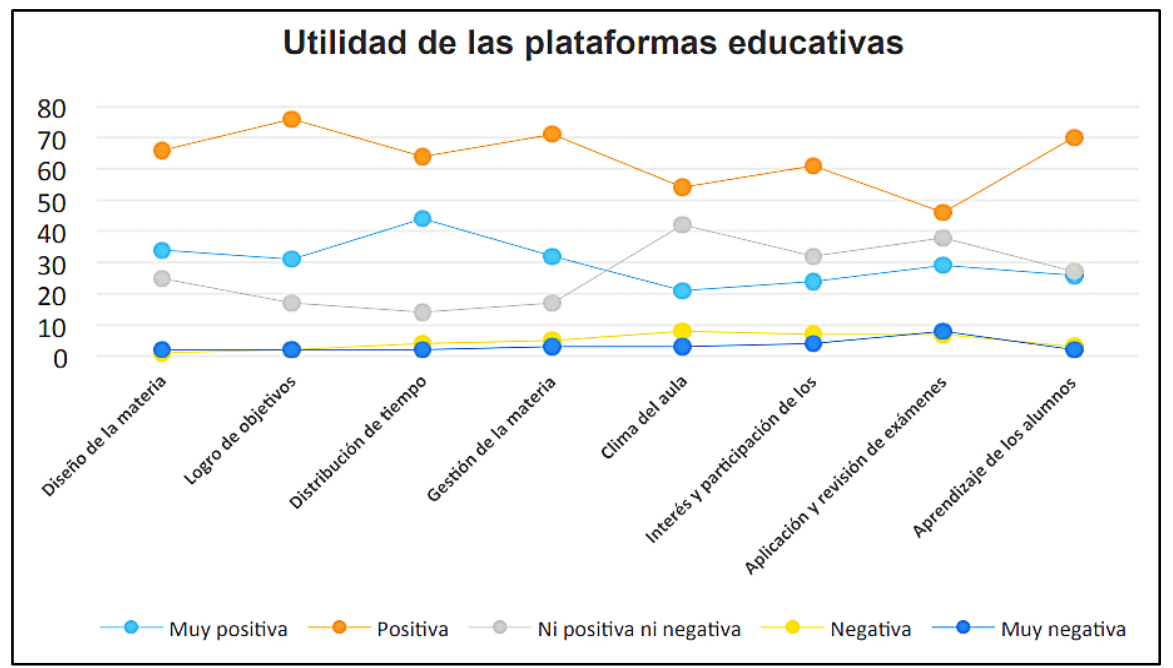

Fuente: file:///C:/Users/RUTH\%20CALO/Downloads/798-2831-1-PB.pdf Elaborado por: Wilmer Ramírez

Los docentes consideran que la utilidad de la plataforma en los ocho aspectos manejados en la encuesta es positiva o muy positiva. Sobresale el parendizaje de los alumnos con el $75 \%$.

Todo esto apoya los resultados realizados a través de la investigación sobre el análisis del impacto académico de la implementación de la plataforma Virtual en el CESUES, 
muestra que el $92 \%$ de los profesores consideraron que la utilización de la Plataforma institucional ha facilitado el contacto con los alumnos, mayor dedicación al preparar clase, gnera mayor actitud positiva en el desarrollo académico.

Tabla N.4: Nivel de uso

\begin{tabular}{|l|c|c|c|c|}
\hline $\begin{array}{c}\text { Nivel de } \\
\text { uso }\end{array}$ & Positivo & Neutro & Negativo & Porcentaje \\
\hline Alto & 56 & 22 & 0 & $38.3 \%$ \\
\hline Medio & 27 & 39 & 11 & $31.3 \%$ \\
\hline Bajo & 16 & 37 & 88 & $30.5 \%$ \\
\hline Total & $\mathbf{9 9}$ & $\mathbf{9 8}$ & $\mathbf{9 9}$ & $\mathbf{1 0 0 . 0 \%}$ \\
\hline
\end{tabular}

Fuente: file://C:/Users/RUTH\%20CALO/Downloads/798-2831-1-PB.pdf Elaborado por: Ruth Calo

Gráfico N.4: Nivel de uso y de impacto de las herramientas tecnológicas.

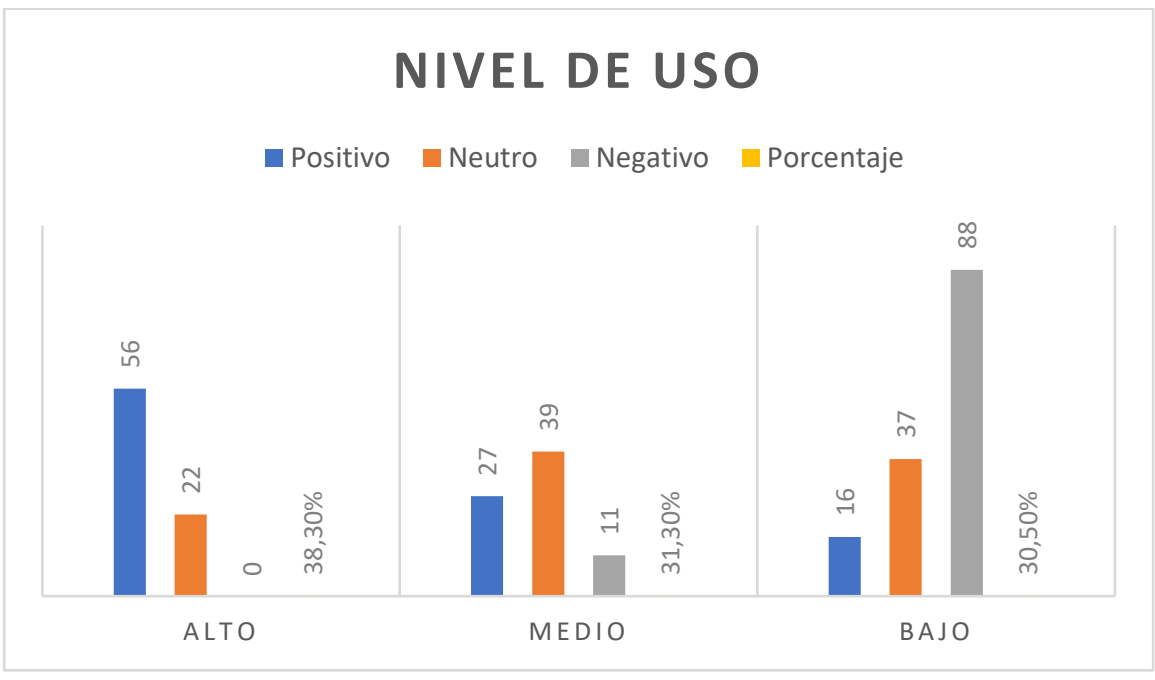

Fuente: file:///C:/Users/RUTH\%20CALO/Downloads/798-2831-1-PB.pdf Elaborado por: Ruth Calo

Se obtuvo que los docentes perciben como herramientas principales y de mayor uso a la distribución de material, encargar actividades individuales, mandar y recibir mensajes, encargar actividades en equipo y retroalimentar y responder dudas; el nivel de uso de las herramientas tecnológicas es alto con un porcentaje de $38.3 \%$, el nivel medio es de $31.3 \%$ y bajo es de $30.5 \%$. 


\section{Conclusiones}

$>$ Mediante fuentes confiables se recopilo información necesaria basada en conceptos entendibles, cada una de las teorías permite al lector tener noción acerca de las Aulas Virtuales en la Educación Superior.

$>$ En la educación virtual el aprendizaje esta centrado en el alumno y su participación activa en la construcción de conocimientos le asegura un aprendizaje significado.

$>$ Las plataformas virtuales son las más usadas en la educación superior, juega un papel relevante en la renovación pedagógica mediante el proceso de enseñanza aprendizaje entre docente y estudiante.

Las plataformas virtuales o entornos de aprendizaje tienen la función de crear, administrar y gestionar de manera más flexible los contenidos académicos a través del internet.

\section{Referencias Bibliográficas}

Abrigo, I. (2015) Aula Virtual una herramienta para dinamizar el proceso de enseñanzaaprendizaje. Riobamba Ecuador. Pág. 12. ISBN: 978-84-608-3627-8.

Aretio, G. (1994) Educación a distancia hoy. España, Madrid UNED. Págs. 10-12. ISBN: 84-362-3129-5

Bernad, E., \& Camacho, M. (2016). La Aula Virtual contenidos y elementos. España: Mc-Graw-Hill Interamericana de España S.L. Págs. 1000. ISBN: 8448612612.

Chamba, L. (2015) Escenarios de Aprendizaje para la inclusión y cohesión social. Universidad de Loja. Ecuador. EDUTEC. Pág. 12. ISBN: 978-84-608-3627-8.

Delgado, M. (2010) Propuesta acerca de un Multimedia - Virtual. Venezuela. Editorial Universitaria. Pág. 6. ISBN: 978-959-16-1305-9.

Domínguez, J. (2013) La Educación a distancia. Chimbote, Perú. Primera Edición. Págs. 352. ISBN: 978-612-46446-0-3

Gallo, R. (2013) Aulas virtuales su integración a la educación presencial. Sembrando experiencias. Uruguay Educa. Pág. 11.

Gómez, L. (2012) Importancia de los programas Virtuales en la Educación Superior. Investigación Educativa. Vol. 15 N²7, 113-126. ISSN: 1728-5852.

Khvilon, E. (2002) Aprendizaje abierto y a distancia. Montevideo, Uruguay. Edición TRILCE. Págs. 11. ISBN: 9974-32-348-7. 
Landa, R. (2010) Diseño Gráfico y publicidad. Fundamentos y soluciones. Editorial: ANAYA MULTIMEDIA. Págs. 496. Lengua: Castellano. ISBN: 9788441528031.

Lepez, L. (2014) La intervención educativa en línea, un dispositivo pedagógico en la formación docente. Buenos Aires, Argentina. Ciencia, Tecnología, Innovación y Educación. Págs. 12. ISBN: 978-84-7666-210-6.

Machado, A. (2004) Educar con Aulas Virtuales. Orientaciones para la innovación en el proceso de enseñanza y aprendizaje. Edición: Primera. Págs. 197. ISBN: 84-7774$147-6$.

Moreno, R. (2014) La Aulas Virtuales en el proceso de enseñanza aprendizaje. Revista Pedagógica Maestro y Sociedad. Santiago Cuba. Vol. 11. N³. Pág. 7. ISSN: 1815-4867.

Porter, L. (1997) Creating the Virtual Classroom: Distance Learning with the Internet. New York, USA. ISBN: 0471178306

Sierra, C. (2012) Educación Virtual aprendizaje autónomo y construcción de conocimiento. Bogotá. Editorial Politécnico Grancolombiano. Págs. 103. ISBN: 978-958-8721-16-3.

Vargas, R. (2004) Perspectivas docentes en la Educación Superior. Transformando la enseñanza en las instituciones de educación superior de la retórica a la realidad virtual. Revista Interinstitucional de Investigación Educativa. México. Vol. 5 $\mathrm{N}^{\circ} 009$. ISSN: 1665-0824.

Zambrano, W. (2012) Modelo de aprendizaje Virtual para la Educación Superior. ECO Ediciones. Bogotá. ISBN: 978-958-648-763-4.

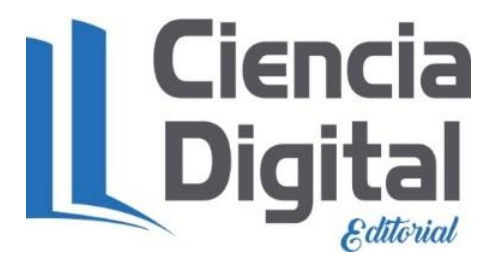




\section{Para citar el artículo indexado.}

Velasteguí P. (2017). Plataformas virtuales y su impacto en la Educación Superior. Revista electrónica Explorador Digital 1(2), 5-21. Recuperado desde:

http://cienciadigital.org/revistacienciadigital2/index.php/exploradordigital/article/view/318/7 30

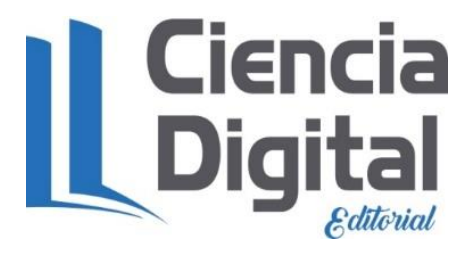

El artículo que se publica es de exclusiva responsabilidad de los autores y no necesariamente reflejan el pensamiento de la Revista Explorador Digital.

El articulo queda en propiedad de la revista y, por tanto, su publicación parcial y/o total en otro medio tiene que ser autorizado por el director o editor de la Revista Explorador Digital.
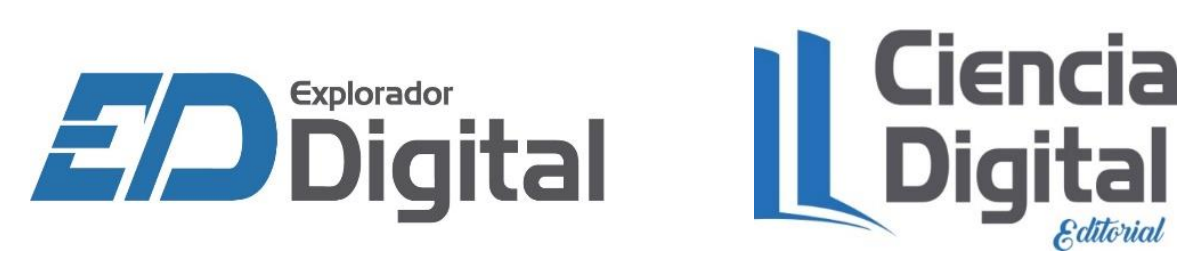